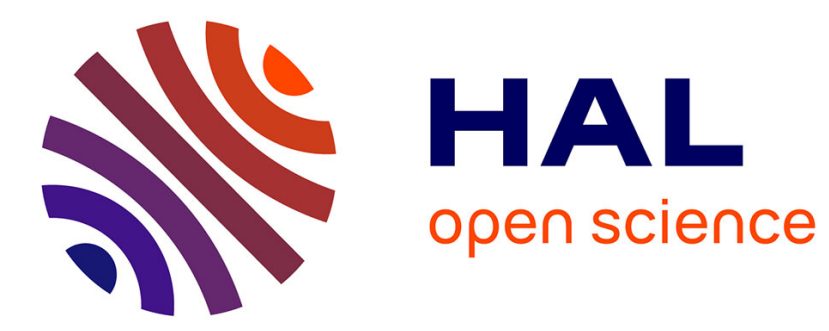

\title{
Gouverner avec le marché: le cas du management commercial de centre-ville
}

\author{
Anais Daniau
}

\section{To cite this version:}

Anais Daniau. Gouverner avec le marché: le cas du management commercial de centre-ville. Revue française de sociologie, 2017, 10.3917/rfs.583.0451 . hal-02462710

\section{HAL Id: hal-02462710 https://hal.science/hal-02462710}

Submitted on 31 Jan 2020

HAL is a multi-disciplinary open access archive for the deposit and dissemination of scientific research documents, whether they are published or not. The documents may come from teaching and research institutions in France or abroad, or from public or private research centers.
L'archive ouverte pluridisciplinaire HAL, est destinée au dépôt et à la diffusion de documents scientifiques de niveau recherche, publiés ou non, émanant des établissements d'enseignement et de recherche français ou étrangers, des laboratoires publics ou privés. 
«Gouverner avec le marché : le cas du management commercial de centre-ville. »

Anaïs Daniau, doctorante,

UMR 5044 CERTOP,

Université Toulouse Jean-Jaurès

anais.daniau@univ-tlse2.fr

Ceci est une pré-version d'un article publié dans la Revue française de sociologie.

La version définitive de cet article : Daniau Anaïs, «Gouverner avec le marché : le cas du management commercial de centre-ville », Revue française de sociologie, 2017/3 (Vol. 58), p. 451-474. DOI : 10.3917/rfs.583.0451. URL : https://www-cairn-info.gorgone.univtoulouse.fr/revue-francaise-de-sociologie-2017-3-page-451.htm

\section{Résumé :}

Face à l'incapacité de l'État à encadrer les implantations commerciales, les municipalités se sont saisies de cette problématique en assurant une partie de la régulation locale. Cet article dépeint la façon dont les acteurs politiques locaux, soutenus par de nouveaux professionnels, ont mis en place de nouvelles politiques publiques de management commercial, en partenariat avec les acteurs économiques. Il interroge les ressorts d'une instrumentation des dynamiques marchandes dans l'action publique locale et questionne les résistances qu'une telle entreprise peut occasionner. À partir du cas d'AchatCommerce, une opération de maîtrise foncière mise en place dans le but de peser sur les implantations commerciales, l'article dépeint les contours d'une forme de gouvernement marchand, dont le succès repose sur la double capacité des gestionnaires locaux à construire la légitimité de leurs politiques publiques par la production de discours neutralisants et à anticiper les comportements et intérêts des acteurs économiques afin d'en tirer parti. 


\section{« Gouverner avec le marché : le cas du management commercial de centre-ville. »}

\section{Introduction.}

Il existe aujourd'hui un large débat en sciences sociales sur la capacité des autorités publiques à gouverner les intérêts privés et à défendre d'autres types de préoccupations. Alors que certains travaux soulignent le rôle croissant des acteurs économiques dans la production et la gestion des espaces publics (Zukin, 1995 ; Davis, 1998) et formulent l'hypothèse de leur privatisation (Häussermann et al. 2008 ; Fleury, 2010), d'autres questionnent la place de ces acteurs au sein de la gouvernance urbaine (Le Galès, 1995 ; Pinson, 2009) et montrent leur capacité à peser sur l'orientation des politiques de la ville (Logan et Molotch, 1987 ; Stone, 1989). S'il est admis que les acteurs de marché participent activement à la fabrique de la ville (Pollard et Halpern, 2013), ce constat est encore plus frappant en ce qui concerne l'activité commerciale qui nous intéresse dans cet article. En effet, la composition commerciale des villes apparait dominée par le jeu d'acteurs économiques poursuivant plus ou moins librement leurs intérêts privés : d'un côté, les propriétaires-bailleurs de locaux commerciaux, de l'autre côté, les commerçants locataires, sans oublier une multitude de professionnels (Dubuisson-Quellier et Cochoy, 2000) engagés dans la conception et la gestion de l'espace commercial urbain: investisseurs, promoteurs, agents immobiliers, notaires, avocats, gestionnaires, experts, développeurs d'enseignes, etc.

Pourtant, depuis plusieurs années, les élus locaux s'investissent plus intensément dans la vie commerciale de leurs territoires. Inquiets face à la multiplication du nombre de commerces vacants, craignant le déclin des espaces centraux, ces derniers se mobilisent et déploient de nouvelles politiques publiques dans le but de redynamiser l'activité commerciale dans les centres villes. Les actions entreprises prennent des formes variées : il peut s'agir d'incitations à se restructurer et se professionnaliser pour les associations de commerçants du territoire, de mesures juridiques visant à encadrer les implantations de commerces, ou encore d'opérations de maîtrise foncière, de procédures plus ou moins formelles de négociation avec les acteurs économiques en place (agents immobiliers, propriétaires de locaux, développeurs d'enseignes, etc.) initiées dans le but de peser sur la composition du paysage commercial. Cette envie grandissante d'intervenir sur l'économie locale, manifeste au vu de la généralisation de telles pratiques, se heurte néanmoins à un enjeu de taille : l'obligation juridique de respecter la liberté d'entreprise. L'action publique doit trouver les moyens de peser sur les comportements économiques sans pour autant les contraindre de façon trop directe (Dubuisson-Quellier et al, 2016).

L'ambition de cet article consiste alors à interroger la place des dynamiques marchandes dans l'action publique en dépeignant la façon dont les acteurs politiques locaux s'en saisissent dans le cadre de leurs politiques publiques. Partant du cas du management commercial des centres villes par les municipalités, nous voudrions décrire une pratique de gouvernement émergente pour laquelle il s'agit de gouverner « avec » le marché, c'est-à-dire par la négociation avec les acteurs économiques, en s'appuyant sur les dynamiques marchandes existantes ou en orientant les comportements économiques en fonction d'objectifs politiques. Le concept d'agencement marchand proposé par Michel Callon (2013) porte en lui cette idée d'une possible ingénierie politique des marchés. Seulement, comme le soulignent les éditeurs du récent numéro de Consumption, Markets and Culture consacré à cette notion (Cochoy, Trompette et Araujo, 2015), le marché a deux sens : celui d'un mode de gouvernement, d'une philosophie, d'une machine marchande, mais aussi celui des «market places », c'est-à-dire des lieux très concrets, diversifiés, qui relèvent de la vie banale et quotidienne. La présente contribution entend combiner ces deux visions, en montrant comment le politique peut s'appuyer sur les comportements marchands ordinaires pour piloter et gouverner le monde collectif dans lequel 
ils prennent place. Il s'agit ici de s'appuyer sur une vision proche de ce que Steve Woolgar et Daniel Neyland (2013) appellent la mundane governance, c'est-à-dire une gouvernance midescendante/mi-ascendante, qui consiste à mobiliser des situations marchandes locales pour obtenir des effets politiques, et inversement à décliner des schémas politiques avec la souplesse du marché afin de les ajuster aux comportements locaux.

La méthodologie utilisée repose sur une étude de cas menée sur la politique de management commercial d'une grande agglomération française combinant plusieurs techniques d'enquête telles que l'observation, l'entretien et l'étude de sources documentaires. L'action publique dans ce domaine est souvent abordée au prisme de ses outils législatifs et juridiques (Mallard, 2015), sans questionner nécessairement l'usage stratégique de ces outils dans la pratique quotidienne, ni même remettre en question le fait que l'action politique ne se limite pas au déploiement de tels outils de cadrage. Ce faisant, notre méthodologie d'enquête s'est voulue proche de l'activité réelle des professionnels chargés de la mise en application de ces outils, notamment de celle du manager de centre-ville, dont le métier est né de la volonté croissante des collectivités locales d'avoir prise sur leur paysage commercial. L'observation que nous avons réalisée entre octobre et décembre 2014 dans le service commerce de la municipalité est très largement inspirée des techniques de shadowing (Czarniawska, 2007) et des parcours commentés (Levy, 2001). Nous avons suivi le manager de centre-ville du service dans son activité quotidienne et recueilli par le biais d'échanges réguliers, ses représentations et justifications (Boltanski et Thevenot, 1991). Il s'agissait de dépasser le simple examen du cadre législatif de l'urbanisme commercial pour offrir une description fine du management commercial en train de se faire, autrement dit, des usages réels des instruments d'urbanisme commercial et des pratiques plus informelles de négociation avec les acteurs politiques et économiques, conformément à notre souci de montrer l'articulation entre pratiques ordinaires et cadrages politiques. Le service où nous avons séjourné nous a également donné accès à des sources documentaires (articles de presse, comptes rendus de réunions, mails archivés, documents de travail, etc.) que nous avons pu mobiliser dans notre étude pour documenter la mise en place du service, celle de la politique publique de management commercial et les différentes étapes de son instrumentation (Halpern et al, 2014). Enfin, plusieurs entretiens semi-directifs menés avec le manager, les techniciens du service, les agents immobiliers ou avec les élus sont venus compléter ces données.

La thèse que nous allons défendre dans cet article est la suivante : les politiques publiques de management commercial se caractérisent à la fois par des modes d'instrumentation souples et par la production de discours neutralisants qui autorisent la poursuite d'objectifs politiques stratégiques. Si à première vue, le management commercial prend les contours d'un «gouvernement doux» mobilisant des outils peu contraignants qui se fondent dans les dynamiques marchandes existantes, les visées des politiques publiques n'en sont pas moins fermes voire violentes pour certaines populations. Dans le cas retenu pour notre étude, les actions menées ont pour fins de repousser certaines catégories de population hors des quartiers centraux et d'amorcer/d'accélérer des processus de gentrification sur certains espaces.

Pour étayer cette thèse, nous organiserons notre exposé en trois parties. Dans un premier temps, nous reviendrons sur les origines des politiques publiques de management commercial, nées à mi-chemin des réalités marchandes et des préoccupations politiques. Cette partie sera l'occasion de souligner le caractère controversé de telles politiques, dont l'existence est sans cesse tendue entre besoin de régulation et respect de l'esprit libéral (I). La partie suivante amorcera la présentation de notre étude de cas en retraçant la mise en place d'une opération de maîtrise foncière intitulée "AchatCommerce » dans l'agglomération de VilledeFrance ${ }^{1}$. Après avoir resitué ce dispositif dans le panel des outils de management commercial déployés sur ce

\footnotetext{
${ }^{1}$ Les lieux, personnes et projets concernés par notre étude sont anonymisés.
} 
territoire, nous nous efforcerons de rendre compte des diverses ambitions politiques ayant motivé la création d'un tel instrument (II). Enfin, la troisième et dernière partie de notre exposé examinera les outils et techniques utilisés par les gestionnaires locaux, notamment par le manager de centre-ville, pour mobiliser le marché comme support de l'action publique, en questionnant par la même occasion, les effets, détournements et résistances que ces dispositifs peuvent occasionner.

\section{Le management commercial, entre libéralisme économique et régulations locales.}

Les pratiques de management commercial émergent à la fin des années 1980 dans un contexte de retour de l'État dans la régulation des implantations commerciales. En effet, la seconde moitié du vingtième siècle fut marquée par l'apparition de nouvelles préoccupations. L'organisation du commerce urbain devient peu à peu un objet de controverse récurrent engageant des acteurs bien au-delà de la sphère marchande (Péron, 1993). À titre d'exemple, la multiplication des complexes commerciaux en lisière de ville tout au long des années 1960 s'est imposée comme l'une des causes majeures de la crise des cœurs urbains, donnant lieu à plusieurs mobilisations remarquables chez les élus, les commerçants et les urbanistes. Plus récemment, les débats autour de la sauvegarde de la « diversité commerciale » (Mallard, 2015, 2016) laissent entendre que le libéralisme économique ne favorise pas nécessairement un développement harmonieux de la ville : uniformisation des paysages urbains due à l'omniprésence d'enseignes internationales, prolifération des activités de service au détriment de commerces de proximité, hyperspécialisation des espaces urbains freinant l'essor d'une activité commerciale diversifiée, voire de la mixité sociale, etc. La multiplication des externalités négatives liées aux localisations commerciales a poussé l'État à générer des outils susceptibles de cadrer, voire de freiner les dynamiques marchandes.

L'urbanisme commercial, défini comme «l'ensemble des processus et des règles, formelles et informelles, qui organisent les activités liées à l'investissement, la construction, la gestion et la réglementation des équipements commerciaux » (Allé, 2015, p. 2), constitue un avatar visible de cette volonté étatique de maîtriser les implantations commerciales et par extension, leurs débordements. Au début des années 1960, les premiers outils d'urbanisme commercial visaient principalement à calibrer l'équipement commercial des banlieues émergentes. La première circulaire interministérielle $n^{\circ} 61-43$ du 25 août 1961 donnait ainsi les premières directives normatives en termes d'équipement commercial des nouveaux quartiers. Mais ces grilles d'équipement furent très rapidement considérées comme obsolètes dans la mesure où elles reproduisaient le sous-équipement commercial des banlieues (Metton, 1978) et se révélaient inefficaces pour traiter les risques de dévitalisation commerciale de centres villes et la désaffection des zones rurales; enfin, elles étaient critiquées par les acteurs économiques (notamment les distributeurs) qui les percevaient comme des entraves à la modernisation commerciale (Péron, 1998). La deuxième circulaire interministérielle du 29 juillet 1969 s'est donnée pour objectif de remédier à ces problèmes en dégageant de nouvelles orientations pour l'urbanisme commercial. Elle prévoit la prise en considération des équipements commerciaux dans les documents d'urbanisme et instaure des comités consultatifs départementaux chargés d'examiner les très gros projets commerciaux (de plus de $10000 \mathrm{~m}^{2}$ ). Le législateur rappelle que «ces directives doivent, bien entendu, répondre aux principes traditionnels de la liberté d'installation et de la concurrence, tout en se conciliant avec les servitudes d'aménagement auxquelles doit obéir la puissance publique $»^{2}$.

\footnotetext{
2 «Circulaire du 29 juillet 1969 relative à la place de l'équipement commercial dans le développement urbain », Journal officiel de la république française, 27 août 1969, p. 08609.
} 
Plusieurs textes législatifs viendront ensuite compléter cette circulaire, dans l'optique de trouver de meilleurs compromis entre ces deux exigences. Sur ce point, la loi d'Orientation du commerce et de l'artisanat (plus connue sous le nom de loi Royer ${ }^{3}$ ) est assez emblématique des problématiques posées par la régulation politique des activités commerciales et surtout des résistances et controverses qu'elle peut susciter. Premièrement, elle n'a pas manqué de provoquer des réactions violentes de la part des distributeurs. Edouard Leclerc s'est mobilisé à plusieurs reprises pour dénoncer l'inflation causée par la loi ou encore la présence de corruption dans les commissions d'urbanisme commercial alors chargées de statuer sur les nouveaux projets de grandes surfaces commerciales. Deuxièmement, les acteurs économiques trouvèrent rapidement des moyens de contourner la réglementation en déposant des projets aux limites des seuils de passage en commission, en les scindant en plusieurs îlots, ou encore en rachetant des surfaces existantes (celles-ci n'étant pas soumises à la procédure d'autorisation). Plusieurs textes de lois à la suite de la loi Royer tenteront de corriger ces défauts, notamment en réduisant le seuil légal de passage en commission à $300 \mathrm{~m}^{2}$ (loi Raffarin de 1996) mais la compatibilité de telles mesures avec les principes de liberté d'entreprise fut remise en cause par la Commission Européenne, obligeant l'Etat français à revoir ses critères de régulation (loi de Modernisation de l'Économie, 2008) : alors que l'octroi des autorisations était majoritairement basé sur des variables économiques, les nouvelles commissions devront justifier leurs décisions par des nécessités d'aménagement du territoire, de développement durable ou de consommation.

\section{Le rapprochement avec l'urbanisme : les municipalités au cour de la régulation.}

Ainsi, face à l'injonction européenne et à l'inefficacité des commissions, plusieurs lois seront votées au long des années 2000 pour faire de l'urbanisme commercial un véritable outil d'aménagement du territoire (Moreno, 2010), au détriment d'un organe de régulation des rapports concurrentiels. Une des évolutions majeures opérées pour tendre vers cette finalité fut d'intégrer les mesures d'urbanisme commercial au sein des instruments traditionnels de l'urbanisme. Ce faisant, ces lois ont largement renforcé le rôle des collectivités locales dans la régulation locale en affirmant leur légitimité à réguler les dynamiques marchandes dans le cadre de projets d'aménagement du territoire. Alors que la loi Dutreil (2005) autorise les municipalités à préempter des baux commerciaux/artisanaux et des fonds de commerce, les lois de Solidarité et de Renouvellement Urbain (dite SRU, 2000), de Modernisation de l'Économie (dite LME, 2008) et Grenelle 2 (2010) permettent d'intégrer des mesures de planification des activités commerciales dans les documents réglementaires d'urbanisme.

Un des enjeux majeurs pour les élus lors de la conception de ces outils consiste à trouver « le bon équilibre » entre régulation du commerce et liberté d'entreprise, à façonner des politiques publiques qui permettent à la fois la poursuite d'objectifs politiques et le respect du dogme marchand. Mais, comme le précisent les juristes Gérard Monédiaire et Dominique Moreno ${ }^{4}$, la forme que les élus locaux souhaitent donner à ce compromis est très différente d'un territoire à l'autre, notamment sur les préoccupations que ces derniers souhaitent mettre en avant dans leurs politiques publiques :

\footnotetext{
${ }^{3}$ Cette loi instaure notamment des commissions départementales d'urbanisme commercial (CDUC), chargées de statuer sur tous les projets commerciaux de plus de $1000 \mathrm{~m}^{2}$. Composées de représentants des consommateurs, de plusieurs élus locaux et professionnels du commerce (la majeure partie du temps, commerçants traditionnels dans le centre-ville), ces commissions servaient principalement à réguler la compétition entre petit et gros commerces et jouaient ainsi le rôle de « police de la concurrence » (Joye, 2007).

${ }^{4}$ Disponible sur le site : http://www.gridauh.fr/comptes-rendus-de-travaux/ecriture-des-plu/
} 
« Il est vrai que les intérêts à concilier sont fort hétéroclites : les uns veulent organiser au mieux le territoire dont ils ont la responsabilité devant leurs électeurs, d'autres veulent avoir le moins de contraintes possibles pour prospérer, d'autres encore veulent bénéficier d'un choix varié d'offres commerciales à proximité... »

À l'instar des politiques procédurales (Lascoumes et Le Bourhis, 1998), les pratiques de management commercial ne suivent pas de modèle unique : elles sont le résultat de bricolages locaux, d'agencements singuliers, déterminés à la fois par les volontés et représentations des acteurs politiques en place et par la manière dont ils envisagent leurs relations aux acteurs économiques. Si la montée en puissance des impératifs de durabilité a permis l'élaboration de nouveaux outils réglementaires destinés à maitriser les implantations commerciales, leur mise en œuvre à l'échelle locale peut dans les faits poursuivre des objectifs relativement diversifiés d'un territoire à un autre (Mallard, 2015). En effet, au-delà des impératifs formels à la durabilité, s'ajoutent aussi depuis les années 1980 des injonctions plus latentes à l'attractivité (Harvey, 1989) et à la compétitivité (Bouinot, 2002). Perçus et construits comme la « vitrine de la ville », les centres villes font depuis ces quarante dernières années l'objet de nombreux investissements matériels et symboliques, analysés par les chercheurs sous le vocable de la gentrification (Bidou-Zachariasen, 2003). Dans ces processus, le commerce joue un rôle important à la fois comme marqueur (Ley, 1966) et catalyseur de ces transformations ${ }^{5}$ (Van Criekingen et Fleury, 2006). Dans un contexte de concurrence urbaine où le commerce participe pleinement à l'identité des villes (Lallement, 2010), les élus locaux prennent conscience de l'intérêt à maîtriser le devenir commercial de leurs cœurs de ville.

Cet intérêt croissant de la part des collectivités locales pour la gestion commerciale de leurs territoires se traduit depuis les années 1990 par un double mouvement de publicisation ${ }^{6}$ et de professionnalisation de cette compétence au sein des municipalités. Que ce soit par le biais d'opérations de gestion foncière telle que Vital'Quartier portée par la SEMAEST ${ }^{7}$, par l'intégration de mesures liées au commerce dans les documents réglementaires d'urbanisme ou encore par la création de services en charge du commerce au sein des municipalités, les élus locaux s'investissent plus intensément dans la vie marchande de leur territoire afin d'en contrôler les dynamiques. L'apparition et le succès récents du métier de manager de centreville s'inscrivent directement dans cette évolution, dans la mesure où ce professionnel est au cœur du développement de ces nouvelles politiques de management commercial. Ces professionnels travaillent en grande majorité pour des collectivités locales où ils reçoivent pour missions la prise en charge des nouveaux instruments d'urbanisme commercial, leur mise en cohérence dans le cadre de stratégies de développement urbain et surtout, leur mise en œuvre par les différents acteurs publics et privés du territoire.

Ce dernier point est crucial dans la mesure où comme nous l'avons souligné, la portée coercitive des outils réglementaires et législatifs d'urbanisme commercial est relativement restreinte pour ne pas mettre en danger la liberté d'entreprise ou le déploiement de la libre concurrence. Néanmoins, ces outils offrent un cadre propice à la négociation avec les acteurs économiques et peuvent être utilisés comme des leviers pour enrôler des protagonistes de la sphère privée dans l'action publique. Cette dernière repose ainsi sur une combinaison subtile d'instruments

\footnotetext{
${ }^{5}$ Si bien que certains chercheurs parlent même de retail gentrification (Bridge et Dowling, 2001 ; Zukin et al, 2009) pour désigner les opérations destinées à requalifier le paysage commercial local dans une optique de gentrification.

${ }^{6}$ La publicisation peut se comprendre comme le fait de «rendre public » un problème défini, mais aussi de revendiquer auprès des pouvoirs publics sa résolution. Jean-Pierre Le Bourhis (2004) définit la publicisation comme le processus par lequel des réponses institutionnelles sont fournies à un problème donné.

${ }^{7}$ La SEMAEST est la Société d'Economie Mixte d'Animation Economique au Service des Territoires de la ville de Paris.
} 
contraignants et d'instruments plus souples, dont l'efficacité repose en partie sur les compétences humaines des gestionnaires chargés de leur mise en oeuvre : le manager de centreville doit montrer des capacités de négociation, des qualités relationnelles et une connaissance solide des problématiques marchandes et politiques. Spécialiste du commerce et de ses dynamiques, il ne limite pas son action à la gestion des externalités négatives du développement de l'appareil marchand mais cherche au contraire, à faire du marché, de ses acteurs et de ses dynamiques, des ressorts possibles pour l'action publique. L'examen d'un instrument de management commercial peut nous permettre de comprendre comment se mêle dans l'usage de ces outils cette pluralité de préoccupations politiques et économiques.

\section{L'opération Achat Commerce : une reprise en main du politique ?}

Après avoir décrit comment les politiques de management commercial fondent leur existence et légitimité sur une politisation du marché des implantations commerciales, nous voudrions maintenant illustrer ce phénomène au travers d'une étude de cas portant sur le dispositif AchatCommerce mis en place par la municipalité de VilledeFrance dans le but de maîtriser les implantations commerciales. Cette opération est intéressante dans la mesure où les édiles locaux ont décidé d'intervenir sur trois quartiers distincts, qui renvoient chacun à une situation spécifique perçue comme problématique par les élus locaux. Ce cas permet alors d'apprécier la souplesse des outils mobilisés ainsi que la grande diversité des motifs qui peuvent conduire une collectivité à intervenir sur le commerce. Nous aurons l'occasion de montrer comment certaines rhétoriques politiques élaborées par les gestionnaires locaux permettent de construire une légitimité aux ambitions de la municipalité. Mais avant d'en venir à la présentation de ce résultat, il convient de resituer le dispositif AchatCommerce dans le plus large panel des instruments de management commercial déployés par VilledeFrance.

\section{Les origines du management commercial à VilledeFrance.}

Avant tout, rappelons que le commerce ne fait pas partie des compétences obligatoires des municipalités. Chaque ville décide à sa guise de prendre en charge cette compétence ou non, avec un degré d'engagement variable en fonction des territoires. Concernant VilledeFrance, la volonté d'intégrer le commerce aux prérogatives municipales s'affirme au milieu des années 2000 avec la création conjointe d'un service commerce et d'un poste de manager de centreville au sein de l'administration municipale. Deux phénomènes semblent être à l'origine de ces créations. Premièrement, la construction d'une nouvelle ligne de métro, perçue par les élus municipaux comme une opportunité de repenser l'organisation du centre-ville dans son ensemble : alors qu'un concours d'urbanistes est lancé pour « redessiner le cœur de ville », la municipalité réfléchit avec les chambres consulaires à la commande d'un diagnostic commercial sur l'espace du centre-ville. En effet, les élus souhaitent que la réhabilitation du centre urbain s'accompagne d'une réflexion sur son attractivité et sur celle de l'activité économique qui s'y déploie. Deuxièmement, le conseiller municipal en charge du commerce et de l'artisanat souhaite donner une plus grande importance à sa délégation en recrutant une équipe de techniciens dédiée à la question du commerce au sein de l'administration municipale

«En 2006, nous avons décidé de créer la mission commerce pour prendre en charge tout ce qui était lié au commerce et à l'artisanat. Je m'étais rendu compte au fil des années que quand on refaisait une rue, quand on faisait des travaux quelque part, il y avait un impact sur le commerce. Et les commerçants n'étaient jamais prévenus, on avait les problèmes qui remontaient après coup. C'était ingérable »-Entretien avec l'adjoint au maire chargé du commerce et de l'artisanat (2002-2008, 2014-2020), juin 2015. 
Conscient du climat de mécontentement chez les commerçants, premières victimes des multiples travaux amenés à fleurir sur le centre urbain, il lance la création d'une mission « commerce et artisanat » chargée d'assurer le lien entre la municipalité, les commerçants et leurs représentants :

«C'est un public à dissocier des riverains et des associations, et la mission du service était de répondre à leurs attentes »-Entretien avec une technicienne du service commerce, décembre 2014.

Le poste de manager de centre-ville est créé à ce moment-là dans le but de «valoriser, dynamiser et d'améliorer l'activité du centre-ville ». Ses missions sont d'accompagner la mise en œuvre du projet VilledeFrance Centre et de venir soutenir l'activité du nouveau service commerce. En plus d'être un interlocuteur privilégié sur la question du commerce et du centreville, il doit aussi assurer la mise en œuvre de la stratégie de développement commercial définie en amont par le comité de pilotage suite à la réalisation du diagnostic ${ }^{8}$. Pour mener à bien ces missions, la fiche de poste diffusée dans le cadre du recrutement précise qu'une formation en « commerce et marketing » serait appréciée pour l'exercice de ce poste, ainsi qu'une bonne « maîtrise des techniques de communication, une connaissance de la culture urbaine et des montages immobiliers ». Pour conclure, le recrutement du manager, tout comme la création du service, répond à un double désir : d'une part, il incarne la volonté du maire de faire du centreville un espace attractif et dynamique, d'asseoir son rôle de centralité métropolitaine ; d'autre part, il permet au conseiller municipal d'apaiser les tensions liées à la réhabilitation du centreville.

Plus d'un an après la création de la mission Commerce et Artisanat, les élections municipales bouleversent l'organisation du service. Après une trentaine d'années aux commandes de la ville, la droite laisse place à une équipe municipale socialiste. Cette nouvelle équipe affiche des ambitions fortes concernant le commerce et souhaite appliquer une politique différente de l'équipe précédente. Alors que l'ancien élu délégué au commerce insistait sur la nécessité de répondre aux demandes des commerçants, la nouvelle équipe municipale envisage plutôt le commerce dans sa dimension urbanistique : on parle moins «d'accompagnement des commerçants » que «d'organisation et d'encadrement » du développement commercial. Dans le discours de la nouvelle adjointe au maire, le commerce se veut un outil d'aménagement du territoire ${ }^{9}$ que la collectivité doit organiser au même titre que le logement ou les transports :

« J'avais toujours tendance à dire : le commerce ne se décrète pas mais il peut s'organiser. Toutes les villes n'ont pas forcément cette vision là, mais c'est vrai que nous on a essayé de mettre en place pendant six ans certains outils pour organiser le commerce. (...) L'ancienne équipe, c'était une vision tout libéral : on laisse faire. Et nous on considère que s'agissant du commerce, la collectivité au nom de l'intérêt général doit l'organiser » - Entretien avec l'adjointe au maire, chargée du commerce, de l'artisanat et du centre-ville (2008-2014), juillet 2014.

\footnotetext{
${ }^{8}$ La fiche de poste publiée par la municipalité en 2006 précise que le manager devra «être à l'écoute des commerçants », « assurer leur relai auprès de la Ville » et enfin, « mobiliser les différents partenaires » que sont la Ville, les chambres consulaires et les associations de commerçants autour de projets communs. Il aura également pour mission de «saisir l'opportunité du réaménagement du centre-ville pour accélérer cette mutation »et de « proposer une stratégie commerciale » et d'assurer sa mise en œuvre. Pour mener à bien cette mission, il pourra «prospecter et favoriser l'implantation de nouvelles enseignes en mobilisant les acteurs économiques et financiers autour des projets », « proposer des projets à vocation commerciale et les mettre en œuvre » et enfin « définir une stratégie de communication et de concertation sur le commerce de centre-ville ».

${ }^{9}$ L'intégration de la mission commerce et artisanat au sein de la direction des opérations d'aménagement est assez révélatrice de cette nouvelle vision.
} 
Sur ce point, il n'est pas impossible que les prédispositions du manager aient tangiblement orienté l'action publique et son instrumentation : ancien cadre salarié du privé et connaisseur des techniques de marketing, ce professionnel fut réellement proactif dans l'accompagnement de son élue à la prise de ses fonctions politiques. Sa capacité à faire des propositions d'actions concrètes puis à les traduire dans un langage politique facilement réappropriable par les élus lui a sans doute permis de se créer une marge d'action non négligeable dans la définition des buts de l'action publique, ainsi que dans celle des moyens pour les atteindre.

Cette montée en puissance de la délégation commerce sur le plan politique a vite trouvé écho sur le plan administratif. Rapidement, le service commerce voit ses effectifs gonfler et ses missions se diversifier avec la mise en place de nouveaux outils destinés à encadrer le développement commercial : l'instauration d'un droit de préemption sur les fonds de commerce et sur les baux commerciaux et artisanaux, l'intégration de plusieurs mesures visant le commerce dans les documents de planification urbaine, la maîtrise de l'offre commerciale sur les zones d'aménagement concertées (ZAC), l'organisation de la concertation avec les acteurs de l'immobilier commercial et enfin, la création d'un observatoire du commerce destiné à suivre l'évolution du paysage commercial.

\section{AchatCommerce : derrière un vocable commun, des enjeux politiques pluriels?}

C'est dans ce contexte politique que naît l'opération AchatCommerce. Au nom du «maintien de la diversité commerciale », le maire et son équipe décident en 2013 de lancer une opération de maîtrise foncière qui consiste pour la Ville à acheter et à rénover des locaux commerciaux par le biais de son Établissement Public Foncier Local (EPFL) pour ensuite les remettre en location auprès de commerçants sélectionnés par la municipalité ${ }^{10}$. De cette manière, les élus espèrent « dynamiser, diversifier, renouveler » le tissu commercial sur trois quartiers concernés par le projet VilledeFrance Centre et par la même occasion soutenir les politiques publiques menées sur ces espaces en termes de sécurité, d'espace public ou encore de logement. L'opération doit contribuer à la «valorisation de trois quartiers clefs » du projet urbain VilledeFrance Centre, en « impact[ant] positivement leur attractivité, la vie du quartier et l'occupation de l'espace public »: LieuA, une place populaire, multiculturelle, en proie à l'insécurité et au «communautarisme »; LieuB, un axe majeur du centre-ville en voie d'être réhabilité et piétonnisé ; et enfin LieuC, le quartier situé entre l'hypercentre et la gare principale de VilledeFrance, dont l'avenir est à nouveau questionné par l'arrivée imminente de la ligne à grande vitesse $(\mathrm{LGV})$. Intégrée à ces projets politiques, l'opération bénéficie ainsi d'une certaine légitimité dans la mesure où les interventions publiques sur le paysage commercial sont motivées par des finalités d'aménagement du territoire :

«C'est-à-dire que pour LieuA, si on n'avait pas eu une volonté un peu globale, on ne serait pas intervenu sur le commerce. L'intérêt de LieuA, c'est la sécurité, la propreté, le logement et le commerce. »-Entretien avec l'adjointe au maire chargée du commerce et de l'artisanat et du quartier centre-ville (2008-2014), juillet 2014.

En outre, le maintien de la diversité commerciale semble s'être imposé dans le débat public comme un objectif politique à part entière depuis le début des années 2000, en atteste son utilisation de plus en plus fréquente dans le langage politique et médiatique. Mais comme le souligne Alexandre Mallard lorsqu'il relate les controverses et débats relatifs à l'emploi de cette expression (Mallard, 2015), celui-ci renvoie à des situations variées qui recouvrent dans les faits des motivations et des modes d'action hétérogènes. Pour les secteurs concernés par

\footnotetext{
${ }^{10}$ Deux agences immobilières sont chargées du recrutement et de la présélection des candidats, mais le choix final reste aux mains des élus.
} 
l'opération AchatCommerce, le constat que nous dressons est que ces espaces cristallisent une multitude de préoccupations que nous allons à présent détailler.

\section{LieuC : soutenir l'émergence d'un nouveau quartier d'affaires.}

LieuC fait l'objet d'enjeux forts par sa situation géographique : situé entre le pôle multimodal de la gare SNCF et l'hypercentre, il constitue un point d'entrée central pour venir à VilledeFrance. Néanmoins, l'arrivée imminente de la Ligne à Grande Vitesse (LGV) a renforcé le caractère stratégique de ce quartier aux yeux des édiles locaux. Cette nouvelle ligne permettrait de rejoindre Paris plus rapidement et les études menées sur l'impact de cette nouvelle infrastructure prévoient une hausse significative du nombre de voyageurs par an. Perçue comme « un atout majeur pour le développement de la métropole mais aussi pour son rayonnement », la LGV conduit la collectivité à saisir cette opportunité en lançant un nouveau projet urbain sur le quartier de la gare. Les rues du secteur LieuC font alors l'objet d'une attention toute particulière. Situées aux abords de la gare que les élus souhaitent restructurer en « nouveau quartier de vie et de travail », elles assurent le lien avec l'hypercentre et par conséquent avec le projet VilledeFrance Centre.

Dans ce cadre, l'opération AchatCommerce doit accompagner la transformation commerciale du quartier dont l'image est plutôt négative selon le diagnostic du service commerce : outre le climat d'insécurité et l'insalubrité qui y règnent, l'offre commerciale est "peu qualitative », marquée par la présence de nombreux sexshops, de clubs, de bars nocturnes et de racolage. La zone est également touchée par une vacance commerciale importante, due en partie à l'état délabré de certains commerces. Le rachat public de cellules commerciales doit ainsi permettre d'installer de nouveaux commerces, plus propres et plus «qualitatifs », en adéquation avec l'identité souhaitée pour le quartier :

« Là on travaille sur l'arrivée du TGV. Il faut l'anticiper donc on est en veille et dès qu'on peut, on rachète des boutiques pour faire muter des sexshops, des commerces de nuit, les commerces qu'on trouve habituellement autour des gares historiques, pour que le jour où le TGV arrive, il n'y ait pas que les propriétaires privés qui profitent de cette arrivée du TGV mais que nous aussi on puisse accueillir de nouveaux locataires et avoir de belles boutiques. » - Entretien avec le manager de centre-ville, mai 2014.

\section{LieuB : favoriser de nouveaux usages.}

Dans le cadre du projet VilledeFrance Centre, les élus souhaitent revaloriser les berges du fleuve en favorisant la liaison avec l'hypercentre. L'axe LieuB, qui constitue aujourd'hui un de ces accès privilégiés aux berges du fleuve, est alors complètement réhabilité : les espaces publics et les règles de circulation sont repensés pour privilégier la circulation des piétons et des vélos. Le manager espère que de telles transformations pourront « encourager la vocation commerciale » de la rue et favoriser une montée en gamme des commerces en place. En effet, le quartier connaît une fréquentation nocturne intense et l'offre commerciale de l'axe LieuB s'est largement adaptée aux usages des étudiants fêtards qui la fréquentent une fois la nuit tombée : épiceries de nuit et échoppes de restauration rapide parsèment les rues. La mutation de l'offre commerciale a donc pour objectif d'apaiser l'activité nocturne et d'impulser de nouveaux usages diurnes et familiaux.

Dans ce cas, on pourrait questionner l'emploi du terme de « diversité commerciale » au regard de la composition effective de la rue. En effet, le relevé commercial effectué par le service sur la rue en 2009 indique que si LieuB est marqué par une forte activité de restauration rapide, le quartier n'est pas pour autant livré à la monoactivité commerciale. Au contraire, la diversité d'activités qui s'y déploie est plutôt remarquable : boutique d'alimentaire bio, phone shop, 
pharmacie, salon de thé, superette, tatoueur, tabac-presse, coiffeurs, agences immobilières, bars, brasserie, boutiques de chaussures, d'accessoires et de prêt à porter, magasin de meubles, libraires, restaurants, magasin de jeux et d'instruments de musique, magasin de photographie, reprographie, fleuriste, hôtel, droguerie. La gamme des produits y est tout aussi variable puisqu'on y trouve à la fois des boutiques de mode à bas prix et des boutiques de créateurs, de la restauration traditionnelle et des fastfood. Ainsi, pour LieuB, c'est une transformation des commerces en place qui est visée et non leur diversification. Par ailleurs, un nouveau relevé commercial effectué un an après la réhabilitation de la rue montre que l'évolution récente de la rue tend plutôt vers un appauvrissement de la diversité commerciale et vers un maintien des activités de restauration.

\section{LieuA : contrôler la mixité sociale, assurer la sécurité de l'espace public.}

Quartier d'immigration, historiquement populaire, multiculturel, LieuA se fait aussi le théâtre d'une forte activité associative et culturelle, qui lui confère une dimension alternative dans l'espace de consommation du centre-ville. Récemment, plusieurs problèmes ont été soulevés par les médias, les habitants et une partie des commerçants quant à l'évolution de ce quartier : mixité sociale en déclin, soucis de propreté, trafics de cigarettes et de drogues, sentiment d'insécurité croissant, nuisances sonores, masculinisation de l'espace public, dégradation des logements et vacance commerciale. Tel est le diagnostic du manager de centre-ville et de son service qui regrettent le «manque de diversité commerciale » sur le quartier :

«LieuA : $16 \%$ de taux de vacance. Centre-ville : 3\%. Il y a un problème. C'est une première alerte. Ensuite, le pourcentage d'activités alimentaires est de $75 \%$. Boucherie, sandwicherie, restaurant, boucherie, sandwicherie, restaurant, boucherie, sandwicherie, restaurant... on sait qu'on a dépassé très largement. Parce qu'on a des grandes valeurs. Vous savez que pour qu'un quartier fonctionne, il y a une sorte de plan de merchandisage : il faut qu'il y ait un boulanger, un épicier, un boucher, une superette, un banque ou deux, un distributeur de billets, un pressing, une laverie...» - Entretien avec le manager de centre-ville, mai 2014.

Alors que certains commerces de proximité quittent la place, d'autres sont mobilisés pour les trafics et n'assurent plus leur activité commerciale d'origine. La municipalité juge alors nécessaire d'intervenir sur le tissu commercial pour remédier aux problèmes de sécurité. L'objectif est double : d'une part, déloger les locataires des commerces à l'origine des trafics et des nuisances ; d'autre part, réintégrer des «commerces de proximité » et des commerces culturels puisque le manager de centre-ville souhaiterait « renforcer l'identité du quartier » en développant une thématique commerciale de type «culture, loisirs, alternatif » qui faciliterait « la lecture du territoire par les consommateurs et les enseignes ».

Une autre ambition de la Ville consiste à enrayer la progressive « radicalisation » religieuse du quartier et le manque de «mixité sociale » (ou devrait-on dire ethnique) sur l'espace public :

« Donc là, ça nous permet de nous rendre compte qu'il y a une thématisation très forte sur le commerce ethnique. Aujourd'hui, quand tous les bazars sur LieuA vendaient jusque-là des plats à tajine et des robes à froufrou pour les petites filles dans l'esprit un peu mauresque, maintenant, il y a des mannequins devant sur le trottoir avec des burqas intégrales » - Entretien avec le manager de centre-ville, mai 2014.

Traiter de la problématique du commerce ethnique reste une question délicate pour les acteurs politiques locaux, notamment dans la justification des choix politiques qui seront faits lors de l'acquisition des cellules commerciales. Sur ce point, il est intéressant de voir comment cette question fut traitée par les techniciens : impossible à désigner directement dans les études officielles, les commerces ethniques sont assimilés à des catégories plus neutres et plus larges 
qui effacent la dimension ethnique (et controversée). À titre d'exemple, une comparaison de relevés commerciaux réalisée par le service commerce (cf. figure 1), utilisée pour justifier l'intervention publique, permet de mettre en évidence une surreprésentation de certains commerces tels que la restauration traditionnelle, la restauration rapide, les coiffeurs, les magasins de meubles/bazar et les boucheries/charcuteries sur LieuA par rapport aux équilibres observés sur l'ensemble de la ville. Ces catégories de commerces représentent évidemment une bonne part des commerces ethniques présents sur le quartier, c'est-à-dire les boucheries halal, les kebabs ou encore les bazars, mais leur désignation et traduction sous des catégories neutres permet d'occulter l'un des objectifs réels de cette politique publique.

\begin{tabular}{|l|c|c|c|c|}
\hline Restauration traditionnelle & 941 & $13,67 \%$ & 24 & $32 \%$ \\
\hline Restauration Rapide & 721 & $10,47 \%$ & 12 & $16 \%$ \\
\hline Coiffeur & 561 & $8,15 \%$ & 7 & $9,33 \%$ \\
\hline $\begin{array}{l}\text { Magasin de } \\
\text { meubles/bazar/Quincaillerie }\end{array}$ & 102 & $1,50 \%$ & 7 & $9,33 \%$ \\
\hline Boucheries/Charcuteries & 173 & $2,50 \%$ & 6 & $8 \%$ \\
\hline
\end{tabular}

Figure 1. Comparaison effectuée entre VilledeFrance (à gauche) et LieuA (à droite) par le service commerce ; nombre et part des commerces selon l'activité exercée.

\section{Une ambition discrète? Gouverner pour le marché.}

Au regard de ces éléments, l'opération AchatCommerce semble être un bon exemple de la manière dont les acteurs publics peuvent décider d'intervenir sur le marché dans le but de maîtriser les débordements liés à l'activité commerciale, voire d'orienter les dynamiques marchandes à des fins politiques (qu'il ne nous appartient pas évidemment de juger). Mais si de prime abord, les problèmes politiques traités sont assez distincts : le contrôle de la mixité sociale et la sécurité sur LieuA, l'impulsion de nouveaux usages sur LieuB ou l'émergence d'un quartier d'affaires sur LieuC, force est de constater que le management commercial passe toujours par un contrôle plus serré de la fréquentation et des usages sur ces quartiers, ceci dans l'optique de produire de l'attractivité marchande sur ces espaces et d'améliorer leur image pour les visiteurs. Comme le stipule le manager de centre-ville :

«On achète dans des quartiers en devenir. Si on y va, c'est parce que le marché n'y va pas la plupart du temps »- Conversation entre le manager et un commerçant, extrait de l'observation au sein du service commerce, novembre 2014.

Cette phrase est révélatrice de la nature de l'opération AchatCommerce. D'un côté, l'action publique construit sa légitimité sur une critique des mécanismes marchands et sur une mise en évidence des impacts négatifs qu'ils produisent sur la vie locale. De l'autre côté, elle se pense aussi pleinement en rapport au marché et à ses dynamiques. L'objectif est bien que le marché « revienne », autrement dit que l'activité économique et la consommation se redéployent sur ces espaces : sur lieuB, le manager souhaite encourager la venue d'une nouvelle catégorie de consommateurs; sur lieuA, l'identité historique du quartier qui est refaçonnée à des fins de marketing territorial ; enfin sur lieuC, le projet de restructuration du quartier de la gare doit clairement encourager l'émergence d'un nouveau pôle de consommation dans la ville.

Au regard de ces éléments, on peut questionner le rôle des managers de centre-ville dans ces phénomènes d'économisation de la ville ${ }^{11}$. Leur sensibilité aux techniques de marketing

\footnotetext{
${ }^{11}$ Même si le métier de manager de centre-ville est aujourd'hui assez largement développé puisqu'on trouve près de 200 professionnels sur l'hexagone, cette profession se déploie surtout sur les villes moyennes et reste rare sur les métropoles (souvent dotées de services commerce qui remplissent des fonctions similaires). Si ce point pourrait poser la question de la généralisation de nos résultats, on observe que les préoccupations d'attractivité occupent également une place importante dans l'activité des managers de villes moyennes.
} 
territorial, leur aptitude à traduire des propositions techniques en projets politiques facilement ré-appropriables par les élus, leur capacité à formuler des enjeux dans un vocabulaire spécifique qui évince les résistances possibles (Staler, 2006) participent à faciliter la mise en œuvre et la réception des politiques de compétitivité et d'attractivité urbaine. Ces politiques, sont pour la plupart pensées au détriment des usagers en place, qu'il s'agisse des habitants ou des commerçants. Perçues comme indésirables pour l'image de la ville, les catégories populaires, qu'il s'agisse des étudiants (lieuB), des prostituées (lieuC) ou encore des familles issues de l'immigration (lieuA) sont en effet les premières visées par ces mesures. Pourtant, l'usage de rhétoriques floues et/ou de catégories neutres rend difficile l'identification de ces objectifs, autorisant ainsi l'introduction discrète de préoccupations d'attractivité marchande dans les politiques publiques déployées. Par ailleurs, un travail similaire de traduction est opéré du côté des acteurs économiques susceptibles de bénéficier de ces politiques. Les interactions avec ces derniers sont soigneusement cadrées et pensées de manière à réorganiser à la marge les dynamiques marchandes sur ces espaces.

\section{L'instrumentation politique des dynamiques marchandes : incitations, cadrages et détournements.}

Une autre façon de réduire le caractère controversé de l'intervention publique réside dans le choix d'instruments souples qui n'entrent pas en contradiction avec le dogme marchand. Sur ce point, l'opération AchatCommerce vient pallier les lacunes des outils d'urbanisme commercial qui étaient jusqu'ici déployés par la municipalité de VilledeFrance. D'un côté, le Plan Local d'Urbanisme (PLU) ne pouvait donner de directives précises quant à la destination des cellules commerciales sans prendre le risque de contraindre significativement l'initiative privée. De l'autre côté, le droit de préemption commercial autorise la municipalité à intervenir sur des transactions en cours pour acquérir des baux commerciaux et en choisir à sa guise les repreneurs mais c'est un droit complexe à mettre en œuvre et peu de collectivités s'y risquent au vu des controverses qu'il suscite. La maitrise foncière des cellules commerciales apparaît alors comme un bon compromis dans la mesure où elle offre à la municipalité la possibilité de maîtriser la destination commerciale de certaines boutiques sans s'immiscer dans les affaires privées, ni contraindre trop fermement la liberté des acteurs économiques.

Au contraire, le marché, ses acteurs et ses dynamiques y sont mobilisés comme des ressorts de l'action publique. Comme le signale Pierre François : "le marché peut constituer un mode d'instrumentation particulier d'une action publique : si l'on admet que le marché est une forme sociale singulière, cette forme peut être investie, délibérément ou de facto, par des collectivités publiques pour mettre en œuvre leurs actions » (François, 2007 : 633). De ce point de vue, l'opération AchatCommerce prend plutôt les contours d'un "gouvernement marchand », qui ne relève pas seulement du partenariat avec les acteurs marchands. En effet, la participation des acteurs économiques à l'action publique ne se pense pas uniquement sous le mode volontaire du contrat (Gaudin, 1999) : elle peut également prendre la forme d'une participation indirecte dès lors que les gestionnaires publics parient sur le comportement futur des agents économiques pour mener à bien leurs politiques publiques. Cette dernière partie sera dédiée à la description de cette forme d'instrumentation politique particulière (Halpern et al, 2014), au travers du cas de l'opération AchatCommerce.

\section{Acquérir des locaux commerciaux : entre négociations et détournements.}

La première phase de l'opération consiste avant tout pour la municipalité à devenir un acteur sur le marché local en se portant acquéreuse de cellules pour ensuite les relouer au même titre que certains propriétaires privés à des porteurs de projets triés sur le volet. Depuis le début de l'opération en 2013, la municipalité dédie un budget d'un million d'euros par an pour cette 
opération et a pu à ce titre, acquérir une dizaine de cellules commerciales, 8 sur LieuA et 2 sur LieuC. Sur LieuB, la réhabilitation des rues avait déjà encouragé la mutation des commerces en place. Si la transformation de la rue n'avait pas pour seul objectif la montée en gamme des commerces environnants, les élus avaient pleinement conscience des conséquences probables de cette rénovation sur le comportement futur des agents économiques :

«On le voit en fait, dès qu'on réaménage de façon qualitative l'espace public, déjà les gens se réapproprient l'espace et du coup les commerces mutent. Ça mute car les commerçants ont davantage envie de s'installer là car les facteurs de commercialité sont meilleurs. »-Entretien avec l'adjointe au maire, chargée du commerce, de l'artisanat et du centre-ville (2008-2014), juillet 2014.

Le manager de centre-ville prend le dossier et le regarde. Il commente : « tu vois, c'est symptomatique de la qualité de la rue ! On n'a pas doublé mais pas loin (en parlant du prix du pas de porte). C'est chouette. »-Commentaire du manager en regardant une DIA sur LieuB, extrait du carnet de terrain, observation au service commerce, novembre 2014.

La réhabilitation de la rue, en même temps qu'elle améliore l'attractivité marchande de la rue encourage une augmentation des loyers et des pas de porte qui rendra certainement difficile l'installation de commerçants moins dotés financièrement. De fait, la Ville n'a pas ressenti le besoin d'acquérir de boutiques sur ce quartier puisque la transformation des espaces publics a suffi à provoquer la mutation des commerces.

Sur LieuA, le choix des locaux à acquérir en priorité se réalise en fonction de plusieurs critères. L'occupation du local commercial constitue évidemment un critère majeur : les locaux vacants (qui nuisent à l'attractivité) et les activités produisant des nuisances (vente d'alcool illicite, plaintes déposées au commissariat, commerces supportant les trafics, pas en règle auprès de la police administrative) sont considérés comme prioritaires à l'acquisition. La synergie possible avec des actions menées sur le logement rentre également dans le calcul puisque la municipalité rachète dans le même temps certains immeubles dans le quartier. Enfin, un facteur important concerne la facilité (ou non) pour les pouvoirs locaux de négocier avec les propriétaires et les locataires des cellules commerciales. À titre d'exemple, acheter un local vide est intéressant dans la mesure où le bail pourra directement être remis en location, contrairement à un local occupé qui obligera la Ville à attendre la fin du bail ou/et à évincer le locataire.

Pour ces raisons, l'EPFL et le service commerce sont en veille constante de façon à guetter des opportunités de négociation : la vente d'un fonds de commerce, d'un local commercial ou d'un immeuble sont autant de chances pour la collectivité de réaliser son intervention. Ainsi, le contact récurrent avec les différents acteurs du quartier (propriétaires, commerçants, agents immobiliers, services municipaux, etc.) a une importance cruciale pour repérer et saisir ces fenêtres d'interventions :

« Ca se fait en fonction du réseau, on est en alerte sur des DIA, on reçoit des porteurs de projet, des vendeurs, des acheteurs... c'est pas facile mais on a un pouvoir d'orientation (...) Je suis en contact avec un certain nombre de propriétaires pour éviter qu'ils vendent à n'importe qui. Un propriétaire qui a un local commercial peut le vendre à tout moment, il faut qu'il fasse attention à qui il le vend, et qu'il soit en accord avec la stratégie de la collectivité »-Entretien avec le chef du service commerce, janvier 2016.

Comme stipulé dans cet entretien, la construction d'un réseau d'acteurs constitue une part cruciale du travail du manager de centre-ville. À ce titre, les diverses autorisations délivrées par la municipalité au moment de l'installation ou de la rénovation d'une boutique commerciale 
(changement d'enseigne, permis de construire, déclaration d'intention d'aliéner) se révèlent être des moments d'action privilégiés pour entrer en contact avec les acteurs économiques du quartier et offrent des leviers d'action intéressants pour négocier avec eux. Dans une logique « win-win », il s'agit de proposer des facilités aux acteurs participant à la politique municipale. À titre d'exemple, les agents immobiliers qui suivent le « plan de merchandisage » imaginé par le service commerce et contribuent à alerter sur les transactions jugées indésirables sont remerciés par des informations qui possèdent une valeur économique pour ces acteurs (avancement de futurs projets d'aménagement, comptages piétons effectués par la municipalité). Du côté de l'administration publique, un des enjeux majeurs du manager de centre-ville consiste à se placer en interlocuteur incontournable sur les questions de commerce et de centre-ville, à se faire connaître des différents services et à collecter le maximum d'informations sur « ce qui se passe » dans le centre-ville. L'observation menée révèle qu'une grande partie du manager de centre-ville est dédiée à la gestion de la boîte mail et au tri des messages reçus. Dans le cas étudié, celui-ci a demandé à être en copie des mails dès lors que cela concerne le centre-ville de VilledeFrance. Les mails «pour information », émanant des différents services, constituent une part importante de sa messagerie et rendent compte de l'important travail relationnel effectué en amont du travail de négociation.

Les compétences de négociation sont au premier plan dans les politiques de management commercial. Pour encourager les propriétaires à coopérer, les techniciens mobilisent une pluralité de ressources de diverses natures : rhétoriques, quand ils soulignent l'intérêt des propriétaires à laisser la collectivité améliorer l'attractivité du quartier et ainsi augmenter la valeur de leur bien immobilier ; relationnelles et symboliques, quand ils sollicitent des figures d'autorité dans leur réseau ; ou encore organisationnelles, quand ils jouent sur la rigidité et la souplesse administratives (accélérer des procédures ou augmenter les contrôles de police administrative par exemple) pour peser sur le comportement des acteurs économiques :

«Quand un porteur de projet ou un propriétaire est reçu par l'élu ou par un responsable administratif de la mairie, de suite, ça n'a pas le même écho. Parce qu'il y a une fonction administrative officielle qui se présente à eux, déjà. Ils savent très bien que pour beaucoup de choses ils ont besoin de la mairie, pour des déclarations, etc. Donc ils savent qu'il vaut mieux travailler avec et dans le sens de la mairie »-Entretien avec le chef du service commerce, janvier 2016.

Ainsi, peuvent-ils inciter des propriétaires à intégrer la stratégie municipale en acceptant de vendre leurs locaux à l'EPFL ou en étant sensibles dans leurs choix de location et de vente, aux orientations prises par la municipalité. Néanmoins, ce dispositif possède également ses failles et ses effets pervers. Premièrement, les acteurs économiques peuvent s'en saisir pour tenter de se sortir de situations économiques difficiles. Certains propriétaires ou commerçants peuvent en effet déclarer de fausses ventes alarmantes dans le but de pousser la municipalité à préempter leur bien, qu'ils n'arrivent pas à céder sur les circuits marchands classiques. Deuxièmement, les ressources mobilisées par les services peuvent faire défaut, notamment durant la période post-élections municipales, où les techniciens ne peuvent pas toujours compter sur un soutien politique effectif. Durant ce temps de fragilité organisationnelle, les acteurs économiques n'hésitent pas à profiter de ces failles pour tenter de faire passer des projets anciennement refusés. Ainsi, au lieu d'être un moyen de créer de l'attractivité sur le quartier, ce dispositif peut être saisi par certains acteurs économiques comme une voie pour quitter le quartier plus facilement.

La commercialisation des boutiques : une politisation des acteurs économiques. 
La commercialisation des cellules acquises constitue le second volet de l'opération. Au vu de la faible attractivité du quartier, la Ville propose des conditions économiques spécifiques pour séduire de potentiels candidats à l'installation et ainsi pallier le manque d'attrait économique du quartier. Une fois acquises, les boutiques sont totalement refaites et les baux rédigés par la municipalité offrent des conditions plus attractives que celles des propriétaires privés pour favoriser l'arrivée de nouveaux types de commerces :

«On fait des travaux pour les remettre en conformité, pour qu'elles soient attractives et ensuite on va commencer à les commercialiser avec des conditions attractives. Sousentendu : on sait que vous n'avez pas nécessairement envie de venir ouvrir des boutiques sur LieuA mais au moins si vous venez, c'est qu'il y a des conditions financières attractives. » - Entretien avec le manager de centre-ville, mai 2014.

Néanmoins, la municipalité subit l'attractivité de son dispositif. Bon nombre d'acteurs qui ne correspondent pas forcément au projet de la municipalité se portent candidats pour intégrer ces boutiques : des associations en recherche de locaux peu onéreux, des commerçants qui ne trouvent pas leur bonheur du côté de propriétaires privés ou encore des commerces déjà présents sur le quartier dont la mairie ne souhaite pas la présence (ex : kebabs). En outre, la lourdeur et la longueur des procédures administratives peuvent décourager les porteurs de projet, malgré des conditions économiques attractives (mais pas si attractives que cela juge l'agent immobilier en charge du dossier). Les agents immobiliers en charge de la commercialisation de ces cellules peinent ainsi à trouver des candidats qui correspondent aux critères des élus et doivent effectuer un tri important dans les porteurs de projet. Le manager de centre-ville en tant qu'interlocuteur privilégié auprès des commerçants réalise également à son niveau une partie de ce travail de filtrage, comme avec ce commerçant souhaitant s'installer dans un local municipal mais dont l'activité ne correspond pas :

« Votre activité ne convient pas. Je dis ça de manière objective, ce n'est pas du délit de faciès. Je ne fais que suivre la stratégie municipale. Par exemple sur le quartier X., si les élus souhaitaient de l'alimentaire de qualité, je refuserais un sexshop. Ca n'a pas de sens dans ce quartier $»-$ Conversation entre le manager de centre-ville et un commerçant, extrait de l'observation dans le service commerce, novembre 2014.

Les refus de la collectivité sont ainsi justifiés au regard de la stratégie politique établie sur le quartier. Mais les acteurs économiques peuvent eux-mêmes mobiliser cette stratégie pour pousser les acteurs politiques à s'intéresser à leur cas individuel et intervenir ainsi en leur faveur

«La communauté d'agglomération de VilledeFrance vient d'acquérir les murs de l'immeuble, suite à l'exercice d'un droit de préemption. Je viens de passer des moments difficiles, pour des raisons personnelles et de santé et j'ai décidé de céder mon fonds de commerce. Comme vous le savez, le quartier LieuA est un quartier difficile, étant donné les formes de délinquance et d'incivisme permanents. Malgré les sollicitations diverses (dont plusieurs propositions autour de 45000 euros), je ne souhaite pas céder mon bail à n'importe qui pour des activités professionnelles qui seraient de nature à accroître les problèmes du quartier » - Extrait d'un courrier d'un commerçant reçu par le service commerce à propos du quartier LieuA.

Ce commerçant mêle dans son discours une pluralité de registres de justification (Boltanski et Thevenot, 1991) : il mobilise à la fois des préoccupations économiques individuelles (ne pas réussir à céder son bail commercial) et des préoccupations politiques plus collectives (le devenir du quartier) et assoit ainsi la légitimité de sa demande en liant son destin individuel aux préoccupations de la municipalité. Cette hybridation des registres de justification se retrouve également dans le discours des porteurs de projets souhaitant s'installer sur le quartier. Si ceux- 
ci doivent attester de la solidité financière et économique de leur projet, ils ont aussi tout intérêt à mettre en avant leur attachement au quartier, leur volonté de participer à son animation et de contribuer au projet politique mené sur le secteur :

«Citoyen de ma ville et de mon quartier, je ne suis pas loyal qu'envers mes clients, mais je suis également acteur de mon voisinage (repas de quartier, bonne ambiance, entraide, etc.). Je connais bien le quartier LieuA, ayant été bénévole sur la place, et j'ai toujours été coutumier des quartiers «populaires » comme celui-ci, ma boutique précédente étant dans le quartier D. de VilledeFrance » - Extrait d'une lettre de motivation reçue par le service commerce à propos du quartier LieuA.

L'action publique présentée ici se rapproche du modèle des marchés concernés tels qu'ils sont définis par la sociologie économique (Geiger et al, 2015), c'est à dire des marchés capables d'ordonner en leur sein des préoccupations à la fois politiques, sociales, et économiques. La présence des pouvoirs publics sur le quartier, le tri des candidats entrants sur le quartier, les interactions constantes entre le service commerce et les acteurs économiques participent à intégrer les préoccupations politiques (notamment d'attractivité) dans le fonctionnement des marchés immobiliers locaux.

\section{Intégrer les dynamiques marchandes à l'action publique.}

La troisième phase de l'opération parie sur l'arrivée de nouveaux acteurs pour réorienter les dynamiques marchandes locales. Élus et techniciens espèrent effectivement que la venue de commerces attractifs dans ces quartiers permettra d'attirer de nouveaux commerces de même standing. Au début de cet article, nous avons évoqué le fait que les municipalités déplorent les dynamiques de spatialisation du commerce quand elles impliquent la multiplication de certaines formes de commerce (banques, fastfood, téléphonie mobile, services, etc.). Néanmoins, elles peuvent aussi utiliser ces dynamiques à leur avantage et prendre en compte le fait que les acteurs économiques soient sensibles au mouvement de leurs concurrents (White, 2002) pour transformer les agencements marchands locaux. Encore faut-il bien connaître les logiques marchandes auxquelles on se confronte car les stratégies d'implantation et les comportements des acteurs économiques sont différents d'un secteur d'activité à l'autre. C'est ici qu'interviennent les savoirs et compétences des managers de centre-ville, considérés comme experts de ces questions. Par un travail constant de lecture de la presse spécialisée, de veille sur les évolutions commerciales en cours, de maintien d'un contact quotidien avec les enseignes et commerçants, ces professionnels appréhendent les dynamiques et stratégies des acteurs économiques dans le but de les anticiper et d'en tirer parti :

«Par exemple, on sait maintenant que quand on a un opérateur de téléphonie qui s'installe quelque part, on peut être sûr que les autres feront tout pour être à cet endroit aussi. » - Extrait d'entretien avec le manager de centre-ville, mai 2014.

L'action publique est pensée ici comme ponctuelle, dans la mesure où il ne s'agit d'acquérir que quelques locaux stratégiques, ceux les plus à même de changer la dynamique du quartier ; et temporaire, en général quelques années, le temps que les acteurs économiques redéfinissent eux-mêmes l'orientation du marché :

«Quand le marché ne joue pas son rôle, on se substitue au marché ponctuellement et on va faire ce qu'on appelle du portage de boutiques. C'est à dire qu'on va les porter 5, 10 ans, jusqu'à ce que le marché retrouve sa diversité »-Entretien avec le manager de centre-ville, mai 2014.

Pour reprendre les termes de l'adjointe au maire, « la Ville impulse et le marché se régule ensuite tout seul ! ». Ici, la propension des acteurs économiques à poursuivre leur propre intérêt, 
à avoir un comportement économique prévisible est perçue comme une condition essentielle du succès de l'action publique, ce qui peut d'ailleurs faire défaut quand les commerçants n'adoptent pas le comportement prévu. Malgré les conditions d'implantation jugées attractives par la ville, les porteurs de projet ne souhaitent pas forcément s'installer sur ces quartiers. De même, si certains acteurs font le pari de venir s'installer, cela n'engrange pas forcément la dynamique promise d'attractivité et ces acteurs finissent par partir faute d'avoir vu la situation s'améliorer.

\section{Conclusion}

L'examen des origines des politiques de management commercial nous a permis de mettre en évidence le rôle prégnant des villes dans la régulation locale des activités économiques et les spécificités des politiques de management commercial. Tout d'abord cantonné à un rôle d'arbitrage de la concurrence entre le commerce périphérique et le commerce de centre-ville, l'urbanisme commercial a évolué pour répondre aux critiques émises sur l'incapacité et l'illégitimité des pouvoirs publics à réguler les rapports concurrentiels dans la ville. Par leur souplesse et grâce à leur intégration dans les outils de l'urbanisme, les nouveaux instruments d'urbanisme commercial ont autorisé le déploiement de politiques de management commercial à l'échelon des villes. L'ambition de cet article consistait à interroger les ressorts, les conditions et les ressources du déploiement de telles politiques. Un premier point de notre exposé a consisté à comprendre comment l'action publique pouvait mêler préoccupations politiques et économiques dans le but d'asseoir la légitimité de l'intervention politique et ainsi occulter certaines ambitions plus discrètes, voire violentes, de la municipalité telles que la transformation d'espaces urbains à des fins d'attractivité marchande, une gestion resserrée de la mixité ethnique et sociale, voire l'évincement de présences populaire et/ou immigrée sur un quartier, préoccupations alors travesties sous un vocable flou et neutre qui rend difficile leur identification et leur critique.

En outre, l'intégration des dynamiques marchandes dans la mise en œuvre des politiques de management commercial permet d'offrir une nouvelle source de légitimité à l'action publique. En effet, face aux critiques du caractère trop dirigiste des grilles d'équipement commercial ou contraignant des commissions d'urbanisme commercial, les municipalités ont mis au point des instruments moins contraignants qui combinent un contrôle et une veille constante de l'activité économique, des mesures incitatives et des procédures de négociation sensées orienter le comportement des acteurs économiques et le rendre conforme à la stratégie municipale, et enfin, une régulation par le marché des entrants et des sortants sur les quartiers visés par ces opérations. Le comportement des acteurs économiques est pleinement mobilisé comme une ressource pour le gouvernement local : les collectivités locales utilisent les leviers de la concurrence, les stratégies et les intérêts des acteurs économiques pour piloter et transformer les dynamiques marchandes sur certains espaces de la ville et accompagner voire prolonger le travail amorcé par les acteurs politiques sur ces quartiers. Néanmoins, ces politiques affectent « le marché » de façon hétérogène : les acteurs économiques jugés indésirables subissent ces politiques tandis que les acteurs perçus comme attractifs et conformes au projet municipal en tirent bénéfice. Le manager de centre-ville par son expertise, ses compétences de négociateur et de communicant, ses savoirs en urbanisme, en marketing et en commerce, son attrait pour le marketing territorial participe pleinement au déploiement de tels politiques d'attractivité et de gentrification. Néanmoins, cela ne garantit pas pour autant leur succès dans la mesure où ces politiques génèrent également des effets du côté des acteurs du marché tels que de l'opportunisme, de l'imprévisibilité ou encore des résistances, qui peuvent entraver le bon déroulement des opérations. Par ailleurs, la force du pouvoir politique repose aussi sur une forte cohésion organisationnelle au sein de la municipalité. Mais dans notre cas, celle-ci a été 
fortement fragilisée en période de changement politique, provoquant ainsi l'arrêt temporaire du dispositif et donc une rupture dans les dynamiques engagées.

\section{Bibliographie}

Allé C., 2015, « La régulation de l'urbanisme commercial : élus locaux versus promoteurs ? », Métropoles [En ligne], 16.

Bidou-Zachariazsen C. (dir.), 2003, Retours en ville : des processus de "gentrification » urbaine aux politiques de « revitalisation » des centres, Paris, Descartes \& Cie, 267 p.

Bridge G., Dowling R., 2001, « Microgeographies of retailing and gentrification », Australian Geographer, 32, 1, p. 93-107.

Boltanski L., Thevenot L., 1991, De la justification, les économies de la grandeur, Éditions Gallimard.

Bouinot J., 2002, La Ville Compétitive ; les clés de la nouvelle gestion urbaine, Paris, Economica, Coll. «Gestion », 180 p.

Callon M., 2013, «Qu'est-ce qu'un agencement marchand? », in : Callon M. et al., Sociologie des agencements marchands, Presses des Mines, p. 325-440.

Cochoy F., Dubuisson-Quellier S., 2000, «Introduction. Les professionnels du marché : vers une sociologie du travail marchand », Sociologie du travail, 42, 3, p. 359-368.

Cochoy F., Trompette P. and Araujo L., 2016, "From market agencements to market agencing: An introduction", Consumption, Markets and Culture, 19 (1), p. 3-16.

Czarniawska B., 2007, Shadowing: And Other Techniques for Doing Fieldwork in Modern Societies, Copenhagen Business School Press DK, 134 p.

Davis M., 1998, Ecology of Fear : Los Angeles and the Imagination of Disaster, Metropolitan Books, New York.

Dubuisson-Quellier S. (dir.), 2016, Gouverner les conduites, Presses de Sciences Po, 471 p.

Fleury A., 2010, «Public/privé : la (re)distribution des rôles dans la production des espaces publics à Paris et à Berlin », Métropoles [En ligne], 8, mis en ligne le 30 novembre 2010, URL : http://metropoles.revues.org/4346

François P., 2007, «Le marché et le politique. Le rôle de l'action publique dans le développement de la musique ancienne », Revue française de science politique, 57 (5), p. 629649.

Gaudin J.-P., 1999, Gouverner par contrat : l'action publique en question, Presses de Sciences Po, Paris, 233 p.

Geiger S., Harrison D., Kjellberg H., and Mallard A., 2014, Concerned Markets: Economic Ordering for Multiple Values, Cheltenham, UK Edward Elgar Publishing Ltd.

Halpern C., Lascoumes P., Le Galès P. (dir.), 2014, L'instrumentation de l'action publique, Controverses, résistances, effets, Presses de Sciences Po, Coll. «Académique », 350 p.

Harvey D., 1989, «From managerialism to Entrepreneuralism : The transformation in Urban Governance in Late Capitalism », GeografiskaAnnaler. Series B, Human Geography, 71, 1, The Roots of Geographical Change: 1973 to the Present (1989), p. 3-17.

Häussermann H., Läpple D., Siebel W., 2008, Stadtpolitik, Francfort/Main, Edition Suhrkamp. 
Joye J.-F., 2007, «Intégrer l'urbanisme commercial dans l'urbanisme général », Actualité juridique. Droit Administratif, 20, juin 2007, p. 1063-1071.

Lallement E., 2010, «Paris métropole marchande, entre singularité et uniformisation urbaine ? », Quaderni, 73 |2010, p. 65-73.

Lascoumes P., Le Bourhis J.-P., 1998, « Le bien commun comme construit territorial. Identités d'action et procédures », Politix, 11, 42, p. 37-66.

Le Bourhis J.-P., 2004, La publicisation des eaux. Rationalité et politique dans la gestion de l'eau en France (1964-2003), th. science politique, Université Panthéon-Sorbonne - Paris I.

Le Galès P., 1995, «Du gouvernement des villes à la gouvernance urbaine », Revue française de science politique, 45, 1, p. 57-95.

Le Galès P., Thatcher M. (dir.), 1995, Les réseaux de politique publique. Débat autour des policy networks, Paris, L'Harmattan, Coll. « Logiques politiques », 272 p.

Lévy E., 2001, « Saisir l'accessibilité : les trajets-voyageurs à la gare du 11 Nord », in : Grosjean M., Thibaud J.-P. (éds.), L'espace urbain en méthodes, Marseille, Parenthèses, p. 47-62.

Ley D., 1996, The new middle class and the remaking of the central city, Oxford, Oxford University Press.

Logan J. R., Molotch H. L., 1987, Urban Fortunes: The Political Economy of Place, Berkeley, University of California Press, 383 p.

Mallard A., 2015, « Concerning urban consumption: on the construction of market agencements for retail trade », Consumption, Markets and Culture, p. 56-70.

Mallard A., 2016, «Exploring urban controversies on retail diversity. An inquiry into the cosmopolitics of markets in the city », in : Blok A., Farias I. (ed.), Urban Cosmopolitics. Agencements, assemblies, atmospheres, Routledge, p. 85-103.

Metton A., 1978, Le commerce et la ville en banlieue parisienne, th., Paris I.

Moreno D., 2010, « La place du commerce dans l'évolution urbaine », Droit et ville, $\mathrm{n}^{\circ} 69$.

Péron R., 1993, La fin des vitrines. Des temples de la consommation aux usines à vendre, ed. ENS Cachan, Coll. «Sciences sociales ».

Péron R., 1998, «L'urbanisme commercial à la française. Quel agrément ? », Annales de la Recherche Urbaine, p. 5-11.

Pinson G., 2009, Gouverner la ville par projet, urbanisme et gouvernance des villes européennes, Paris, Presses de Sciences Po, 418 p.

Pollard J., Halpern C., 2013, «Les acteurs de marché font-ils la ville ? », EspacesTemps.net, Traverses, 17.06.2013.

Steiner P., Trespeusch M. (dir.), 2014, Marchés contestés. Quand le marché rencontre la morale, Toulouse, Presses Universitaires du Midi.

Stone C., 1989, Regime Politics. Governing Atlanta, 1946-86, Lawrence, University of Kansas Press.

Van Criekingen M., Fleury A., 2006, « La ville branchée : gentrification et dynamiques commerciales à Bruxelles et à Paris », Belgeo [En ligne], 1-2|2006, mis en ligne le 27 décembre 2013, URL : http://belgeo.revues.org/10950

White H. C., 2002, Markets from networks. Socioeconomic models of production, Princeton (NJ), Princeton University Press. 
Woolgar S., Neyland D., 2013, Mundane Governance: Ontology and Accountability. Oxford University Press: Oxford, 282 p.

Zukin S., 1995, The Cultures of Cities, Blackwell, New York.

Zukin S., Trujillo V., Frase P., Jackson D., Decuber T. Walker A., 2009, « New Retail Capital et Neighborhood Change : Boutiques and Gentrification in New York City », City \& Community, 8, 1, p. 47-64. 